\title{
A catalogue of somatic mutations
}

A comprehensive analysis of somatic (acquired) mutations across exons, introns and intergenic regions in 560 breast cancer genomes is reported in a recent Nature paper. In a second paper, published in Nature Communications, the authors explore how these mutations relate to genome architecture.

Most 'driver' mutations, which confer a proliferative advantage on cells that favours cancer development, are thought to be acquired over time as a result of a range of mutational processes, rather than inherited. The characterization of these somatic mutations by next-generation sequencing offers opportunities to gain insights into the genes and pathways that go awry in cancer. In the first study, Nik-Zainal et al. report the analysis of whole-genome sequences of tumours and normal tissue from 560 breast cancer ( 556 women and 4 men), across non-coding as well as protein-coding regions. Overall, the authors implicate 93 protein-coding genes in breast cancer pathogenesis and characterize a number of mutational signatures (the specific pattern left on the genome by each mutational process). These signatures included 12 base substitutions and 6 genome rearrangements, 3 of which could be linked to defective homologousrecombination-based DNA repair. Dominantly acting activated fusion genes and non-coding driver mutations were found to be rare.

In the second paper, Morganella et al. used the same 560 breast cancer genomes to investigate the effects of genomic architecture on mutational signatures. By analysing the genomic distribution of mutations as mutational signatures, the authors were able to infer how a number of cellular processes and features, including transcription, replication and chromatin organization, influence mutagenesis in breast tissue. Taken together, the two studies provide insights into the mutational processes underlying the development of breast cancer and their genomic distribution, bringing us one step closer to a more complete understanding of the somatic genetic basis of this disease.

Linda Koch

ORIGINAL ARTICLES Nik-Zainal, S. et al. Landscape of somatic mutations in 560 breast cancer whole-genome sequences. Nature http://dx.doi.org/10.1038/nature17676 (2016)| Morganella, S. et al. The topography of mutational processes in breast cancer genomes. Nat. Commun. 7,11383 (2016)

FURTHER READING Helleday, T., Eshtad, S. \& Nik-Zainal, S. Mechanisms underlying mutational signatures in human cancers. Nat. Rev. Genet. 15, 585-598 (2014) 